\title{
Arrhythmia Risk and Obesity
}

\section{Ioana Mozos*}

Department of Functional Sciences, "Victor Babes" University of Medicine and Pharmacy, Timisoara, Romania

\begin{abstract}
Obesity is a known cardiovascular risk factor and it increases the risk of cardiac arrhythmias and sudden cardiac death. The most commonly reported arrhythmias are atrial fibrillation and ventricular tachycardia.

The present review focuses on the mechanisms linking overweight and obesity with cardiac arrhythmias and provides a brief review of the latest studies in this area.

Obesity is one of the very few identified modifiable risk factors for the occurrence and progression of atrial fibrillation, and the mechanisms linking atrial fibrillation and obesity include: structural and electrophysiological atrial remodeling, metabolic factors, sympatho-vagal imbalance, clinical links (obstructive sleep apnea, cardiovascular comorbidities) and inflammation.

The main mechanisms leading to ventricular arrhythmia and sudden cardiac death in obese individuals include cardiomyopathy, metabolic factors, sympathetic hyperinnervation, obesity-induced electrophysiological remodeling, coronary heart disease as common comorbidity and radical weight reduction strategies.

Electrocardiographic monitoring, including $\mathrm{P}$ wave and QT interval duration, are extremely important in obese patients. Weight control may be an effective strategy for reducing the burden of cardiac arrhythmias and sudden cardiac death.
\end{abstract}

Keywords: Obesity; Cardiac arrhythmias; Atrial fibrillation; Ventricular arrhythmia; QT interval; Late ventricular potentials; Sudden cardiac death; Electrocardiography; Pathophysiology; Body mass index; Obstructive sleep apnea; Inflammation; Oxidative stress

\section{Introduction}

Overweight and obesity are defined, according to the World Health Organization as abnormal or excessive fat accumulation. A person with a body mass index (BMI) between 25 and $29,9 \mathrm{~kg} / \mathrm{m}^{2}$ is considered overweight, and with a BMI of $30 \mathrm{~kg} / \mathrm{m}^{2}$ or more obese [1]. Obesity has reached epidemic proportions, its prevalence continues to increase worldwide and cardiovascular disease dominates the mortality and morbidity in obese patients [2,3]. The most rapidly growing segment of the obese population is the severely obese, with a BMI of $40 \mathrm{~kg} / \mathrm{m}^{2}$ or more [4].

Obesity is a cardiovascular risk factor and is significantly associated with hypertension, coronary heart disease, cardiac arrhythmias, heart failure, diabetes mellitus and dyslipidemia $[2,5,6]$. In obese and overweight individuals, the cardiac structure and function change as a consequence of an increased in total plasma and blood volume, with subsequent increase in left ventricular filling and cardiac output, causing left ventricular hypertrophy, left atrium and right ventricular hypertrophy, and the peripheral resistance is decreased [5,7-9]. Cardiomyopathy of obesity includes increased ventricular mass and chamber size, hypoplastic coronary arteries and severe coronary atherosclerosis [10]. The increased abdominal mass impairs the function of the diaphragm, reducing oxygen supply and enabling arrhythmia and sudden cardiac death [11]. Hypoxia can cause pulmonary vasoconstriction, pulmonary hypertension and right heart failure [11]. Despite higher prevalence of cardiovascular pathology with excessive fat, a survival advantage has been also described in obese patients with heart failure, hypertension, myocardial infarction or peripheral arterial disease: "the obesity paradox" [3,9].

There are multiple mechanisms linking obesity to cardiovascular pathology, including the cardiometabolic consequences of obesity, accelerated atherosclerosis, altered release of adipokines and chemical mediators, promoting a proinflammatory and prothrombotic state, neurohormonal activation with increased sympathetic tone, endothelial dysfunction, increased arterial stiffness, left ventricular hypertrophy, hemodynamic alterations, altered cardiomyocyte electrical properties, obesity-related cardiomyopathy, infiltration of fat into the myocardium and coronary artery calcification $[5,6,8,9,12,13]$.

Obesity increases the risk of arrhythmias. The most commonly noted arrhythmias include sinus arrhythmia, premature atrial and ventricular contractions, atrial fibrillation, ventricular and supraventricular tachycardia [5]. Cardiac arrhythmias may be precipitated in obese by several factors, including: hypoxia, hypercapnia, electrolyte imbalances due to diuretic therapy, coronary heart disease, increased circulating catecholamines, obstructive sleep apnea, left ventricular hypertrophy, and fatty infiltration of the conduction system [2].

The present review focuses on the mechanisms linking overweight and obesity with cardiac arrhythmias and provides a brief review of the latest studies in this area.

\section{Atrial Arrhythmias and Obesity}

\section{Premature atrial contractions}

Premature atrial contractions (PAC) are independent predictors of

*Corresponding author: loana Mozos, M.D., Ph.D., Associate Professor, Department of Functional Sciences, "Victor Babes" University of Medicine and Pharmacy, T. Vladimirescu Str. 14, 300173, Timisoara, Romania, Tel: +40745610004; E-mail: ioanamozos@yahoo.de

Received November 20, 2013; Accepted December 26, 2013; Published January 01,2014

Citation: Mozos I (2014) Arrhythmia Risk and Obesity. J Mol Genet Med S1: 006 doi: 10.4172/1747-0862.S1-006

Copyright: (c) 2014 Mozos I. This is an open-access article distributed under the terms of the Creative Commons Attribution License, which permits unrestricted use, distribution, and reproduction in any medium, provided the original author and source are credited 
atrial fibrillation and stroke, and were associated with several factors, including age, height, history of cardiovascular disease, natriuretic peptide levels, but not body mass index [14]. The metabolic syndrome, a cluster of atherosclerotic risk factors, including central obesity, was associated with an increased number of extrasystoles, supraventricular tachyarrhythmias, sinus node arrest and atrial fibrillation [15-17]. Significant correlations were found between arrhythmias and the number of components of the metabolic syndrome, demonstrating a cause-effect relationship [15].

\section{Sinus arrhythmias}

Sinus arrhythmias are frequent in obese patients, especially sinus bradycardia [18]. Prolonged sinus pauses were revealed by 24-hour ECG monitoring in a patient with Prader-Willi syndrome, requiring pacemaker insertion [19]. The Prader-Willi syndrome is a rare genetic syndrome, resulting from an abnormality in chromosome 15 , and is characterized by severe childhood obesity, short stature, behavioral disturbances, intellectual disability, and severe hyperphagia [19]. The patients are at increased risk of sudden death, and elevated inflammatory markers, premature atherosclerosis, increased systolic blood pressure and abnormal microcirculatory responses, could have an important contribution [19,20].

\section{Atrial fibrillation}

Atrial fibrillation is the most common sustained arrhythmia in clinical practice, and is associated with an increased risk of ischemic stroke, heart failure, systemic embolism, cognitive dysfunction, dementia, diminished quality of life and exercise capacity, and death [16,21-27]. The classic risk factors for atrial fibrillation include hypertension, valvular disease, cardiomyopathy, male sex, aging, thyroid disease and diabetes mellitus [22,28,29]. The increasing incidence of atrial fibrillation is due to advancing age of the population, a better survival of individuals with structural heart disease and the current obesity epidemics [12,25]. Aging increases the risk of developing atrial fibrillation through age-dependent structural and electrophysiological characteristics $[22,30]$. The majority of patients with atrial fibrillation suffer from a cardiovascular disorder (substrate related), which may have an additive effect on the perpetuation of the arrhythmia $[22,26]$, but there are some patients with "lone" atrial fibrillation, without an underlying cardiovascular disorder [28]. Patients with lone atrial fibrillation have normal life expectancy, a low risk of stroke, and rarely progress to persistent or permanent atrial fibrillation [28].

Several studies demonstrated the link between obesity and atrial fibrillation [31,32], but earlier reports did not support this association [33,34].

Wanahita et al. [31] found a risk of developing atrial fibrillation up to $50 \%$ in overweight and obese. The mechanisms linking obesity and atrial fibrillation are complex, including atrial remodeling, increased activity in the conduction system of the heart, electrophysiological remodeling of the left atrium, neurohormonal activation, an increased sympathetic activity, elevated plasma volume, increasing left ventricular diastolic filling pressure, metabolic factors (insulin resistance, dyslipidemia, free fatty acids, reactive oxygen species), mechanical effects (raised intrathoracic pressures and obstructive sleep apnea), increased arterial stiffness, genetic predisposition and geneenvironment interactions $[6,8,16,21,25,27,29,34-37]$ (Figure 1).

Increased left atrial pressure may increase atrial ectopy, triggering atrial fibrillation [36]. Atrial remodeling is characterized by changes in ion channel function, calcium homeostasis, and atrial structure, such

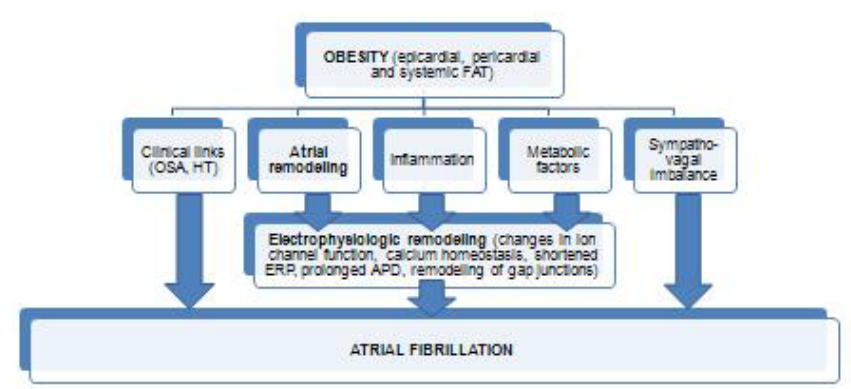

Figure 1: Pathophysiological links between obesity and atrial fibrillation.

as cellular hypertrophy, activation of fibroblasts and subsequent tissue fibrosis, enabling the occurrence of "triggers" for atrial fibrillation and the formation of a "substrate" for atrial fibrillation that promotes its perpetuation [30]. Increased left atrial pressure and volume, shortened effective refractory period in the left atrium and pulmonary vein may facilitate atrial fibrillation in obese patients [38]. Prolonged BMI - mediated left atrial stretch was associated with the development of fibrosis [34]. There are several clinical links between obesity and atrial fibrillation, including hypertension, macro- and microvascular ischemia, impaired coronary perfusion and obstructive sleep apnea [27]. A high fat diet, even in the absence of obesity, increases the sympathetic activity, enabling arrhythmic events [39].

Tsang et al. [34] found obesity as a risk factor for progression of paroxysmal to permanent atrial fibrillation, depending on the left atrial size.

Watanabe et al. [16] demonstrated an increased risk for the development of atrial fibrillation in patients with metabolic syndrome. Most of the components of the metabolic syndrome were related to the development of atrial fibrillation. Inflammation, oxidative and mechanical stress and enlargement of the atrium, loss of muscle mass, fibrosis and spatial remodeling of gap junctions have been proposed as factors linking the components of the metabolic syndrome and atrial fibrillation [16,40-42].

Nicolaou et al. [21] reported a greater risk for progression of atrial fibrillation in obese elderly women due to the enlarged atrium. The combination aging-obesity increases sympathetic activity and angiotensin II production, which increased blood pressure and affects left atrial remodeling and the onset of atrial fibrillation, through the association with left ventricular hypertrophy and subclinical diastolic dysfunction, which affects the size and function of both atria and ventricles [21,27,43-45].

Abed and Wittert [35] mentioned atrial electro-structural dysfunction enabled by obesity-related risk factors, such as hypertension, vascular disease, obstructive sleep apnea, insulin resistance and pericardial fat. Atrial fibrillation risk was associated not only with pericardial, but also with systemic and regional epicardial adiposity [27]. The effect of epicardial fat was independent of BMI and other known risk factors for atrial fibrillation [46].

$\mathrm{Li}$ et al. [26] reported an increased risk of atrial fibrillation in patients with myocardial infarction, left ventricular hypertrophy, obesity and alcohol consumption in a Chinese population, but a lower prevalence compared to European studies.

Atrial fibrillation is the most common arrhythmia in women, as well. Obesity was associated with atrial fibrillation in postmenopausal 
women [47], among fertile young women [25,29], in pregnant women with a history of atrial fibrillation, and in female health care professionals without cardiovascular history [48]. Karasoy et al. [29] reported a strong relationship between obesity and new-onset atrial fibrillation, especially lone atrial fibrillation, in young, fertile women. Hemodynamic changes associated with pregnancy and delivery and electrophysiological phenomena may play an important role in developing lone atrial fibrillation [28,29].

Early life factors may be involved in the pathogenesis of atrial fibrillation and obesity among individuals with a low birth weight $[49,50]$. A significant part of the association between birth weight and atrial fibrillation is mediated through height and cumulative exposure to elevated body mass, and lower birth weight protects the atria against fibrillation in adults [50]. Genetic or environmental intrauterine factors may "program" adult body mass, left atrial size and subsequent atrial fibrillation risk [50]. Genome studies on patients with atrial fibrillation emphasized the importance of genes related to atrial fibrillation, especially PITX2, on chromosome 4q25, for the development of the left atrium, and ZFHX3, in growth regulation of several tissues [50].

Obesity was associated with an increased atrial fibrillation risk in community- and population-based cohort studies, and in cardiothoracic surgery cohorts, independent of type of cardiac surgery $[27,51]$. Body mass index was included, as an atrial fibrillation risk factor, in multiple risk scores, including the Framingham Heart Study 10-year AF risk calculator [27].

The Atrial fibrillation Follow-up Investigation of Rhythm Management Study (AFFIRM) demonstrated better outcomes (lower all-cause and cardiovascular mortality) in obese patients with atrial fibrillation than in lean, concluding that an obesity paradox exists for outcomes in obese patients with atrial fibrillation [52].

$\mathrm{P}$ wave indices, derived from the surface ECG, include P-wave duration, morphology and amplitude, may assess atrial electric function, progressively altered by obesity $[27,53]$. P wave dispersion, the difference between maximum and minimum $\mathrm{P}$ wave duration, an electrocardiographic marker for the prediction of atrial fibrillation, was increased in obese women [53]. The most important electrocardiographic markers of atrial remodeling and prolongation of atrial conduction time are: an increased maximum $\mathrm{P}$ wave duration in the standard, 12-lead electrocardiogram and long signal-averaged $\mathrm{P}$ wave duration [54]

Adipose tissue itself may be directly involved in the pathogenesis of cardiovascular disease, considering that obesity has been associated with generalized enlargement of fat depots, involved in the production of pro-inflammatory cytokines and reactive oxygen species and uncontrolled release of fatty acids [8,55]. Cardiac adiposity is characterized by an increase in intramyocardial triglyceride content and an enlargement of the fat tissue surrounding the heart and vessels, which can lead to myocardial damage [55]. Fatty acid infiltration and overload promotes fatty acid oxidation, accumulation of triglycerides and metabolites which can impair calcium signaling, beta-oxydation and glucose utilization, damage mitochondrial function with increased production of reactive species, proapoptotic and inflammatory molecules [55]. Fatty infiltration or "fatty metamorphosis" can induce abnormal automaticity from degenerated myocardial cells [56].

Several biomarkers have been identified as a link between obesity and atrial fibrillation, including inflammatory markers, adipocytokines, pericardial and epicardial fat, and atrial tissue $[27,57,58]$. Obesity is an established inflammatory condition [59], and adipocytes enable local inflammation through adipocytokines and proinflammatory cytokines [60]. Inflammation, measured by plasma levels of high sensitivity $\mathrm{C}$ reactive protein, fibrinogen and soluble intracellular adhesion molecule-1, was significantly associated with the risk of incident atrial fibrillation in healthy, middle-aged women, free of cardiovascular disease [58]. Various other inflammatory markers have been associated with atrial fibrillation, including tumor necrosis factor alpha, interleukin 2, 6 and 8 [24]. Inflammatory infiltrates, myocyte necrosis, and fibrosis have been found in atrial biopsies of patients with atrial fibrillation [61,62]. Chronic inflammation may induce electrophysiological and structural changes in the atrial myocardium predisposing patients with triggering atrial foci to atrial fibrillation [63]. Proposed mechanisms linking inflammation and atrial fibrillation include endothelial dysfunction, production of tissue factor from monocytes, increased platelet activation, and increased expression of fibrinogen [24]. It is still not clear if inflammatory markers elevation is a consequence or a cause of atrial fibrillation [23,58]. Probably preexisting inflammation initiates the arrhythmia that subsequently propagates an inflammatory response, enabling persistence of atrial fibrillation [23]. C-reactive protein has been shown to decrease cardiac contractility [64]. On the other hand, it was suggested that inflammation is not a major mediator of the atrial fibrillation risk associated with obesity [48].

Adiponectin is one of the adipocytokines secreted by the adipose tissue, both a biomarker and a possibly mediator of cardiovascular disease, with antiatherogenic, antidiabetic and antiinflammatory properties, able to influence the extent of atrial and left ventricular remodeling, which can increase cardiac contractility and action potential duration by inhibiting delayed rectifier potassium currents $[59,60,65]$. Higher adiponectin levels were detected in patients with chronic atrial fibrillation than in paroxysmal atrial fibrillation, correlated with a collagen type I degradation marker, demonstrating that adiponectin is a useful marker of atrial remodeling [65]. Activation of fibroblasts and subsequent fibrosis contribute to atrial structural remodeling and heterogeneity of the cardiac conduction tissue $[56,60,65]$.

Epicardial fat tissue modulates atrial electrophysiological and contractive properties, through inflammatory cytokines, adipocytokines and adipocyte-cardiomyocyte interactions, and heart failure epicardial fat has a greater arrhythmogenic effect on the left atrium, prolonging action potential duration [66]. Epicardial adipose tissue was also suggested to be involved in the maintenance of atrial fibrillation [67]. The right atrium is more resistant to hypoxia/ reoxygenation than the left atrium, due to higher heat shock proteins [68]. Several experiments showed that epicardial adipocytes modulate atrial cardiac ionic currents with decrease of delayed rectifier inward and outward currents and increase of late sodium currents and L-type calcium currents [69].

Future research should focus on the relation between atrial fibrillation and adiposity phenotypes, gene-environment interactions, obesity years, childhood obesity, increased risk due to comorbidities, weight loss, atrial remodeling and reverse remodeling, mechanisms of resolution of atrial fibrillation and new biomarkers [23,27]. Electrocardiographic monitoring is extremely important in obese patients, considering that a substantial proportion of atrial fibrillation episodes are asymptomatic.

\section{Ventricular Arrhythmias and Obesity}

Patients with morbid obesity have high rates of sudden cardiac death (SCD), before the development of heart disease [70-72]. SCD is more common in obese persons than in lean individuals, although 
progression to heart failure may be the most common cause of death in patients with obesity-associated cardiomyopathy $[6,12,13]$. Obesity was an important comorbidity in SCD patients with structurally normal hearts [73]. The main mechanisms leading to arrhythmia and SCD in obese include cardiomyopathy, with myocyte hypertrophy, abnormal cardiomyocyte lipid deposits, lipotoxicity of the myocardium induced by free fatty acids, cardiac fibrosis and mononuclear cell infiltration, and obesity-induced electrophysiological remodeling, myocardial infarction, left ventricular hypertrophy, impaired connexins, sympathetic hyperinnervation and parasympathetic withdrawal [13,7277] (Figure 2). Fatty infiltration separates the myocardial bundles and disrupts their parallel orientation, impairing ventricular activation and resulting in heterogeneous repolarization [72].

Obesity is a known risk factor for coronary heart disease and myocardial infarction, due to the association with other cardiovascular risk factors [78-80]. Ischemia/reperfusion injury enabled scar expansion and inadequate fibrosis in obese rats and mice [74-76].

Feeding rats a high calorie diet resulted not only in increased body weight, visceral fat content, plasma insulin, nonesterified free fatty acids and triglycerides, but also in cardiac hypertrophy [75]. Myocyte hypertrophy may lead to left ventricular hypertrophy in "healthy" obese rats after high-fat diet [7]. The fatty acid milieu causes structural and functional changes in the heart of obese [7].

Hearts of female rats fed a high-fat diet were associated with arrhythmias and an impaired reparative fibrotic response following an ischemic insult, due to sympathetic hyperinnervation and impaired gap junction proteins, despite a nonsignificant increase in body weight, a normal plasma lipid profile [76].

Increased intracellular lipid content can impair repolarization due to a decrease in potassium channel protein levels, causing ventricular tachycardia and sudden cardiac death [13]. Adipocytokines from epicardial fat significantly decrease delayed rectifier outward currents in cardiomyocytes, prolonging action potential duration and facilitating triggered activity with early after depolarizations [81].

Obese patients have an increased frequency of premature ventricular contractions compared to healthy controls, unrelated to hypertension or concentric ventricular hypertrophy $[13,82]$.

Besides changes in $\mathrm{P}$ wave and $\mathrm{PR}$ interval, several other electrocardiographic changes may occur in obese individuals, including an increased heart rate, prolonged QT interval and QRS complex duration, increased QT dispersion, modified QRS voltage, STT abnormalities, multiple electrocardiographic criteria for left

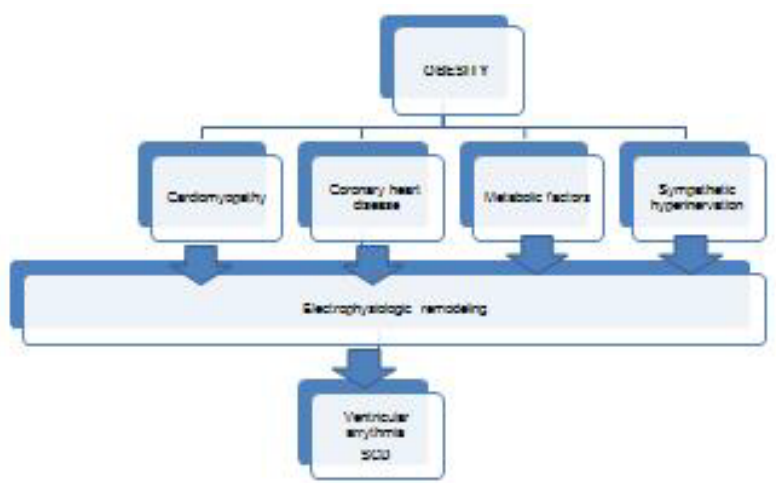

Figure 2: Pathophysiological links between obesity and sudden cardiac death. ventricular hypertrophy, flattening of the $\mathrm{T}$ waves and leftward shifts of the P wave, QRS and T wave axes [4,18,83-85]. Many of these ECG abnormalities are reversible with substantial weight loss [84].

Obese individuals have a faster heart rate and reduced heart rate variability due to abnormalities in sympatho-vagal balance, factors associated with an increased risk of myocardial infarction and sudden cardiac death $[3,12]$.

Therapy of ventricular arrhythmias in obese patients includes ICD and pacemaker implantation, chronic optimal medical therapy, programmed weight reduction [85]. Food-, plant- and drug-based therapies for weight loss have, lately, gained great attention [86]. Several ventricular arrhythmias and SCD were reported due to weight loss pills in young women [86-88]. Sibutramine, an oral anorexiant, prolongs the QT interval and causes a reversible cardiomyopathy, ventricular fibrillation and cardiac arrest, and was withdrawn from the market worldwide $[89,90]$. Potassium loss due to diuretics can prolong the QT interval and cause serious arrhythmias [90]. Clinicians prescribing weight loss pills should consider the cardiovascular profile and monitor their patients for ECG abnormalities.

\section{The QT Interval and QT Dispersion in Obese}

Weight gain delays cardiac repolarization, and several studies reported prolonged QT intervals in obese patients, including persons with uncomplicated obesity and abdominal fat deposition [13,52,9196]. Other studies, conducted in patients with uncomplicated obesity, reported no effect of weight gain on cardiac repolarization [97]. The differences between the outcomes of the studies may be due to heterogeneity of study populations, different QT interval measurement techniques and measurement errors [52].

Prolonged heart rate corrected QT interval (QTc) correlated with body mass index, waist circumference, waist-to-hip ratio, and obese patients with glucose intolerance and hyperinsulinemia are more likely to have a prolonged QT interval $[93,94,96,98]$. The correlation between QTc and waist circumference was stronger than the one between QTc and body mass index, indicating that abdominal obesity is a more important predictor of cardiac risk than BMI in obese patients [96]. The prolonged QT interval was associated with an increased sympathetic and decreased parasympathetic tone in obese patients [6]. Insulin resistance and decreased serum HDL cholesterol were risk factors for QTc prolongation in obese children [99]. Patients with uncomplicated metabolic syndrome had a greater dispersion of ventricular repolarization time and increased maximal and minimal QTc [100].

The QTc interval correlated with free fatty acid level in normal subjects and obese women, and fatty infiltration can increase dispersions of action potential duration, increasing the possibility of reentry circuits $[81,93,101]$. Corbi et al. [93] found a significant relationship between QTc intervals, waist-to-hip ratio, plasma free fatty acids, epinephrine and norepinephrine concentrations, suggesting autonomic nervous system dysfunction as a possible mechanism of the prolonged QTc intervals in visceral obesity. Elevated plasma free fatty acid level has a stimulatory effect on the sympathetic nervous system [102]. QT dispersion in nonapneic simple snoring, overweight adults was significantly increased [95]. An increased QT dispersion in obese women was associated with left ventricular hypertrophy and late ventricular potentials [103].

QT interval prolongation in obese patients is related to the functional and structural changes in the heart, metabolic factors 
including hyperinsulinemia, glucose intolerance, free fatty acids, decreased HDL cholesterol, autonomic dysfunction, fatty infiltration and comorbidities $[52,93,99,101]$.

Body mass index did not affect overall QT duration, but QT dispersion was significantly higher in normal-weight coronary patients than in obese [98]. On the other hand, intraventricular conduction was affected in obese patients with an old myocardial infarction [11].

Reduction of body weight in obese women resulted in significant shortening of the QTc interval and QT dispersion, correlated with reduction in plasma free fatty acid concentration [93] and regression of left ventricular hypertrophy [104]. Shortening of the QT interval and cardiac parasympathetic activity increase, may reduce the risk of potentially fatal arrhythmias and sudden death [93]. Sleeve gastrectomy in morbidly obese patients was associated with QT interval shortening 3 months after surgery [105].

QT prolongation, cardiac arrhythmias and sudden deaths have been reported among dieters using very low energy diets, recommending regular inpatient electrocardiographic monitoring for obese patients on therapeutic starvation [106].

\section{Late Ventricular Potentials in Obese}

Late ventricular potentials are high frequency, low amplitude signals, appearing in the terminal part of the QRS complex, obtained using signal averaged ECG [77]. According to an international convention, late ventricular potentials are present, if 2 of the following criteria are positive: SAECG-QRS duration $>120 \mathrm{~ms}$, low amplitude signal: LAS40 (duration of the terminal part of the QRS complex with an amplitude below $40 \mu \mathrm{V}>38 \mathrm{~ms}$, and the root mean square signal amplitude of the last $40 \mathrm{~ms}$ of the signal $<20 \mu \mathrm{V}$ (RMS40) [107]

Several studies reported late ventricular potentials in obese patients $[72,92,108]$. The presence of late ventricular potentials in obese individuals could be secondary to changes in obesity-associated cardiomyopathy, including fibrosis, fat and mononuclear cell infiltration and myocyte hypertrophy $[12,109]$.

Late ventricular potentials are affected by body size and left ventricular mass [108]. Masui et al. [108] reported no correlation between QRS duration and root mean square voltage with body weight and body mass index, sum of skin folds or left ventricular mass. Positive linear correlations were found between low amplitude signals and weight, body mass index, sum of skin folds [108]. Subadipose tissue may prolong low amplitude signals by attenuation of the QRS complex, suggesting that low amplitude signals are inappropriate for the definition of positive late ventricular potentials in obese persons [108].

Lalani et al. [72] considered pericardial adipose tissue, fibrosis and myocyte hypertrophy to be related with the increased frequency of late ventricular potentials in obese individuals, and body mass index as an independent predictor of abnormal signal averaged ECG results.

Body mass index correlated with SAECG-QRS duration and LAS40 in patients with an old myocardial infarction, suggesting a body mass index dependent sudden cardiac death risk [110].

\section{Cardiac Conduction System Involvement in Obese}

Frank et al reported unfrequent conduction abnormalities in obese patients [18]. Involvement of the conduction system in sudden death of obese young people has been explored by Bharati et al, reporting an hypertrophied and enlarged heart, focal mononuclear cells in and around the sinoatrial node, marked fat throughout the conduction system, fibrosis of the atrioventricular bundle and left bundle branch, a fibrous atrioventricular node and focal fibrosis of the ventricular septum [111]. The mild to moderately obese patients demonstrated a higher degree of fibrosis compared to the markedly obese, showing a higher amount of fat [111].

\section{Obstructive Sleep Apnea and Arrhythmias in Obese Patients}

Obstructive sleep apnea (OSA) is defined by recurrent episodes of upper airway collapse during sleep, impairing ventilation and resulting in subsequent hypoxia, hypercapnia, sleep arousals and loud snoring [53,112]. Overnight polysomnography is the gold standard test to diagnose and stratify sleep apnea, but patients may be screened for OSA with a simple tool, as well, such as Berlin questionnaire [113]. Central obesity is a common finding and major risk factor for obstructive sleep apnea and increased adipose tissue predisposes to airway narrowing [6,112]. The major risk factors for OSA are, besides obesity, age, male gender and alterations in craniofacial structure [114].

OSA is a risk factor for excessive daytime sleepiness, road traffic accidents, cardiovascular morbidity (hypertension, stroke, metabolic syndrome) and mortality [112]. Both tachyarrhythmia and bradyarrhythmia are possible causes of cardiovascular morbidity in patients with OSA [114]. OSA is also independently associated with type 2 diabetes mellitus, insulin resistance, diabetic microvascular complications, dyslipidemia and bronchial asthma [115].

Increased mortality in patients with OSA, particularly at night, emphasizes the importance of identifying the mechanisms of myocardial electrical instability [115]. The main mechanisms linking OSA with cardiac arrhythmias are: increased intrathoracic pressure, autonomic imbalance (repetitive oscillations between sympathetic and parasympathetic predominance), systemic and pulmonary hypertension, structural and electrical atrial and ventricular remodeling, inflammation, diastolic dysfunction, endothelial dysfunction, atherogenesis and myocardial ischemia, night-time hypoxemia and acidosis [22,27,28,53,114-117]. Repetitive inspiration against collapsed upper airways generates important shifts in intrathoracic pressure, transmitted to the atria and contributing to atrial remodeling (enlargement and fibrosis) [53]. Hypoxemia may cause pulmonary vasoconstriction and pulmonary hypertension and stimulates the sympathetic nervous system via reflex mechanisms [28,118]. The combination of repetitive fluctuations in heart rate, blood pressure and intrathoracic pressure during sleep with increased sympathetic tone, leads to increased left and right ventricular wall stress, left and right ventricular hypertrophy and systolic and diastolic heart failure [118]. Several observations point toward a long-lasting sympathetic hyperactivity, affected by comorbidities [119].

All kinds of arrhythmias have been observed in patients with OSA, ranging from asymptomatic sinus bradycardia to fatal ventricular arrhythmias and asystole [112,114]. A paroxysm of atrial fibrillation may lead to central sleep apnea, due to an acute decrease in left ventricular filling, with an increase in pulmonary wedge pressure and consequent stimulation of pulmonary vagal receptors [28]. Both OSA and central sleep apnea modulate the autonomic nervous system at night through central respiratory-cardiac coupling in the brainstem, chemoreflexes, baroreflexes, and reflexes related to lung inflation and arousals [120]. The repetitive oscillations between sympathetic and parasympathetic predominance enable the development of cardiac arrhythmias: bradyarrhythmias when parasympathetic 
tone predominates, and atrial and ventricular arrhythmias when sympathetic tone predominates [118].

Studies on the prevalence of bradyarrhythmias in patients with OSA have yielded conflicting results [118]. Many studies reported sleep-related bradyarrhythmias or asystole in patients with OSA, not related to a diseased sinus node or atrio-ventricular conduction system $[112,121]$. Uninhibited paroxysmal parasympathetic discharges lead to marked paroxysmal bradycardia [113]. Bradyarrhythmias during sleep are associated with OSA severity, and concomitant chronic obstructive pulmonary disease or beta 2-therapy may play a role in development of tachyarrhythmias [117]. Other studies reported no increase in bradyarrhythmias rates $[122,123]$.

Atrial remodeling related to OSA may cause atrial arrhythmias, including interatrial block, atrial fibrillation and flutter. It has been reported that OSA may increase left atrial size independently of obesity, hypertension and diastolic dysfunction [118]. OSA was found as an independent risk factor for atrial fibrillation [113]. The association between atrial fibrillation prevalence and OSA is related to the severity of hypoxemia [124], chronically increased sympathetic activity due to hypoxemia and hypercapnia, surges in adrenergic and vagal tone, atrial remodeling, and a higher prevalence of traditional risk factors [113,118]. Nocturnal hypoxemia may cause mitochondrial dysfunction, resulting in repetitive oxidative stress and increased production of inflammatory cytokines, leading to endothelial dysfunction, insulin resistance, hypercoagulability and adverse myocardial remodeling [113]. The increased sympathetic tone and intrathoracic pressure may lower atrial effective refractory period due to activation of acetylcholine-dependent potassium channels, enabling pulmonary vein discharges and atrial dilation [113]. The high prevalence of atrial fibrillation explains the increased risk of stroke and heart failure in patients with OSA [112].

OSA not only promotes initiation of atrial fibrillation, but also may contribute to its progression, and makes management of atrial fibrillation more difficult $[113,125]$. OSA was associated with higher rates of early and overall recurrence after catheter ablation of atrial fibrillation and it predicts atrial fibrillation recurrence after pulmonary vein isolation [113,125-127]. A significantly higher incidence of atrial fibrillation recurrences following cardioversion and ablation was found in patients with untreated compared to treated OSA, suggesting that OSA may trigger atrial fibrillation [128]. Serum markers of oxidative stress and free radical production predict atrial fibrillation recurrences after atrial fibrillation ablation [113]. It is important for physicians to monitor atrial fibrillation patients for OSA and monitor those with OSA for atrial fibrillation [125].

A significant association between OSA and ventricular arrhythmias has been also demonstrated, due to myocardial ischemia, the procoagulant, proinflammatory and heightened adrenergic state, heart remodeling, systolic and diastolic heart failure, arterial desaturation and arousal $[118,129]$. Ventricular ectopy and nonsustained ventricular tachycardia were recorded during sleep, but the prognosis of nonsustained ventricular tachycardia is debatable in the absence of heart disease [130]. A high prevalence of OSA was also found in implantable cardioverter-defibrillator recipients [53]. Namtvedt et al. [117] reported an increased prevalence of ventricular premature complexes in middle-aged patients with mild or moderate OSA. A high prevalence of sleep-disordered breathing (60\%) was found in patients with ventricular arrhythmias and normal left ventricular function, demonstrating a strong association in patients without heart failure [129]. Repetitive intermittent hypoxemia and hypercapnia due to OSA, act through chemoreceptors, increase sympathetic activity and induce ventricular arrhythmias [129]. Only few studies investigated the association between OSA and sudden cardiac death. Habitual snorers and patients with OSA had a higher risk of sudden cardiac death in the early morning $[131,132]$.

Obstructive sleep apnea is increasing in the pediatric population, as well, and is more common among overweight and obese boys [114]. QT dispersion was significantly increased only in obese children with OSA [114]. QT dispersion increases also in adult patients with moderate-to-severe OSA, and a positive correlation was found between QT dispersion and the severity of OSA [133].

Patients who presented advanced atrioventricular block had more severe OSA than those without arrhythmias [134].

OSA is a common but underestimated disorder, linked to cardiovascular morbidity and mortality. Unfortunately, OSA is largely undiagnosed, due to the insufficient awareness of the disorder among physicians and there should be heightened suspicion of OSA in patients with atrial fibrillation $[112,113]$. The deleterious cardiovascular effects of OSA may be reversible with early therapy [114], and patients could benefit of weight normalization, ECG monitoring, continuous positive airway pressure ventilation (CPAP) and avoidance of factors known to increase upper airway obstruction, such as alcohol consumption (alcohol reduces the muscle tone of the upper airways and prologs apnea) and use of sedatives [112,117]. CPAP may improve oxidative stress and reduce the structural and electrical remodeling of the atria due to OSA, resulting in a lower atrial fibrillation recurrence rate $[113,127]$. On the other hand, CPAP reduces preload, which may further compromise diastolic filling of the ventricles, already impaired to the loss of atrial pump in atrial fibrillation [113]. More attention should be given to patients with more severe OSA or concomitant comorbidities [117].

\section{Oxidative Stress and Arrhythmogenesis}

Obese subjects exhibit increased systemic oxidative stress, enhanced by abdominal adiposity and associated with adiponectin deficiency [135]. Recent clinical and experimental evidence demonstrated the involvement of oxidative stress in cardiac electrical and structural remodeling $[23,136,137]$. Reactive oxygen species may impair $\mathrm{Na}$, $\mathrm{K}$ and $\mathrm{Ca}$ channels, Na-Ca exchanger activity, may be implicated in gap junction remodeling, decrease the action potential amplitude and duration, and increase the incidence of cardiac arrhythmias in animal models [136-140].

Oxidative stress results in decreased hERG protein levels, accelerated activation and deactivation of hERG, and increase in current amplitude of hERG and hKv1.5, allowing a greater amount of $\mathrm{K}$ ions to flow through these channels in the phase 3 of the action potential, downregulation of Ito (responsible for the rapid repolarization phase), and increases the channel opening probability of Ik1 (inward rectifying channel) [136,137].

An abundance of oxidative markers was found in atrial tissues from patients with persistent atrial fibrillation, although plasma markers of oxidative stress did not correlate with developing atrial fibrillation [23]. Not only L-type calcium channels and sodium channels are sensitive to the redox state, but also cardiac creatine kinase, explaining the link between inflammation and the structural remodeling of the atrium [23].

General and mitochondrial anti-oxidants may be promising therapeutic strategies of arrhythmias in obese patients [137]. 


\section{Conclusions}

The present review illustrates the significant association of excess body weight with an increased arrhythmia risk, and the mechanisms explaining the high prevalence of atrial and ventricular arrhythmias in overweight and obese patients, with implications for prevention and therapy of atrial fibrillation and sudden cardiac death. Atrial fibrillation is very common in obese patients with cardiovascular disorders. Obesity impairs ventricular depolarization and repolarization, prolongs the QT interval and is frequently associated with ventricular arrhythmias, even before the development of heart disease.

Obstructive sleep apnea is common in obese patients and it is linked to atrial and ventricular arrhythmias due to structural and electrical cardiac remodeling, an increased sympathetic tone, systemic and pulmonary hypertension, intermittent hypoxia and inflammation, activation of humoral, metabolic and thrombotic factors.

Obesity is one of the very few identified modifiable risk factors for arrhythmias, and the development of a risk score, incorporating clinical, biochemical, ECG and genetic obesity markers would be useful in assessing arrhythmia risk. Considering the multiple factors linking obesity and cardiac arrhythmias, may provide a more comprehensive picture of arrhythmia risk, enabling preventive and therapeutic measures. Future research should focus on the genetic component enabling the association obesity-cardiac arrhythmias, changes in the molecular components of the ion channels and repolarization reserve, nontraditional obesity biomarkers predicting arrhythmia risk, without neglecting the use of other known ECG markers.

Weight control may be a reasonable strategy for reducing the burden of cardiac arrhythmia, but electrocardiographic monitoring is recommended for obese patients during weight reduction programs. Prevention and treatment of obesity and detection and control of obstructive sleep apnea could represent cornerstones for the prevention of cardiac arrhythmias. Standard 12-lead ECG can play an important role in obese patients in predicting atrial fibrillation (P wave duration and dispersion) and ventricular arrhythmia risk (QT interval duration and dispersion). ECG is the gold standard for diagnosis of arrhythmias, considering that most of the patients are asymptomatic.

\section{References}

1. [Noauthors listed] (2000) Obesity: preventing and managing the global epidemic. Report of a WHO consultation. World Health Organ Tech Rep Ser 894: i-xii, 1-253.

2. Adams JP, Murphy PG (2000) Obesity in anesthesia and intensive care. $\mathrm{Br}$ Anaest 85: 95-108.

3. Lavie CJ, Milani RV, Ventura HO (2009) Obesity and cardiovascular disease: risk factor, paradox, and impact of weight loss. J Am Coll Cardiol 53: 19251932.

4. Poirier P, Eckel RH (2008) Cardiovascular consequences of obesity. Drug Discovery Today: Therapeutic strategies 5: 45-51.

5. Wilborn C, Beckham J, Campbell B, Harvey T, Galbreath M, et al. (2005) Obesity: prevalence, theories, medical consequences, management, and research directions. J Int Soc Sports Nutr 2: 4-31.

6. Diaz-Melean CM, Somers VK, Rodriguez-Escudero JP, Singh P, Sochor O, et al. (2013) Mechanisms of adverse cardiometabolic consequences of obesity. Curr Atheroscler Rep 15: 364

7. Jeckel KM, Miller KE, Chicco AJ, Chapman PL, Mulligan CM, et al. (2011) The role of dietary fatty acids in predicting myocardial structure in fat-fed rats. Lipids Health Dis 10: 92.

8. Chrostowska M, Szyndler A, Hoffmann M, Narkiewicz K (2013) Impact of obesity on cardiovascular health. Best Pract Res Clin Endocrinol Metab 27:
$147-156$.

9. Guglin M, Baxi K, Schabath M (2013) Anatomy of the obesity paradox in heart failure. Heart Fail Rev.

10. Alexander JK (1985) The cardiomyopathy of obesity. Prog Cardiovasc Dis $27:$ 325-334

11. Mozos I (2005) Chronic myocardial infarction. Influence of obesity on dispersion parameters. Timisoara Medical Journal 55: 272-275.

12. López-Jiménez F, Cortés-Bergoderi M (2011) Update: systemic diseases and the cardiovascular system (i): obesity and the heart. Rev Esp Cardiol 64: 140-149.

13. Huang H, Amin V, Gurin M, Wan E, Thorp E, et al. (2013) Diet-induced obesity causes long QT and reduces transcription of voltage-gated potassium channels. J Mol Cell Cardiol 59: 151-158.

14. Conen D, Adam M, Roche F, Barthelemy JC, Felber Dietrich D, et al. (2012) Premature atrial contractions in the general population: frequency and risk factors. Circulation 126: 2302-2308.

15. Provotorov VM, GlukhovskiÄ $\neg$ ML (2009) [Rhythm and conductivity disorders in patients at the initial stages of metabolic syndrome]. Klin Med (Mosk) 87: 26-28.

16. Watanabe H, Tanabe N, Watanabe T, Darbar D, Roden DM, et al. (2008) Metabolic syndrome and risk of development of atrial fibrillation: the Niigata preventive medicine study. Circulation 117: 1255-1260.

17. Alberti KGMM, Eckel RH, Grundy SM, Zimmet PZ, Cleeman JI, et al. (2009) Harmonizing the Metabolic Syndrome: A Joint Interim Statement of the International Diabetes Federation Task Force on Epidemiology and Prevention; National Heart, Lung and Blood Institute; American Heart Association; World Heart Federation; International Atherosclerosis Society; and International Association for the Study of Obesity. Circulation 120: 1640-1645.

18. Frank S, Colliver JA, FrankA (1986) The electrocardiogram in obesity: statistical analysis of 1,029 patients. J Am Coll Cardiol 7: 295-299.

19. Patel S, Harmer JA, Loughnan G, Skilton MR, Steinbeck K, et al. (2007) Characteristics of cardiac and vascular structure and function in Prader-Will syndrome. Clin Endocrinol (Oxf) 66: 771-777.

20. Lamb AS, Johnson WM (1987) Premature coronary artery atherosclerosis in a patient with Prader-Willi syndrome. Am J Med Genet 28: 873-880.

21. Nicolaou VN, Papadakis JE, Dermitzakis G, Dermitzaki SI, Tsakiris AK (2009) Effect of obesity on atrial size in older women with non-valvular paroxysmal atrial fibrillation. Aging Clin Exp Res 21: 344-348.

22. European Heart Rhythm Association; European Association for Cardio-Thoracic Surgery, Camm AJ, Kirchhof P, Lip GY, Schotten U, et al. (2010) Guidelines for the management of atrial fibrillation: the Task Force for the Management of Atrial fibrillation of the European Society of Cardiology (ESC). Eur Heart J 31 : 2369-2429.

23. Endoh Y, Endoh I, Geczy C, Nakagomi A, Kusama Y, et al. (2011) Inflammation and atrial fibrillation. J Arrhythmia 27:106-115.

24. Guo Y, Lip GY, Apostolakis S (2012) Inflammation in atrial fibrillation. J Am Coll Cardiol 60: 2263-2270.

25. Conen D (2013) Obesity and atrial fibrillation: the evidence is gaining weight Europace 15: 771-772.

26. Li Y, Wu YF, Chen KP, Li X, Zhang X, et al. (2013) Prevalence of atrial fibrillation in China and its risk factors. Biomed Environ Sci 26: 709-716.

27. Magnani JW, Hylek EM, Apovian CM (2013) Obesity begets atrial fibrillation: a contemporary summary. Circulation 128: 401-405.

28. Schoonderwoerd BA, Smit MD, Pen L, Van Gelder IC (2008) New risk factors for atrial fibrillation: causes of 'not-so-lone atrial fibrillation'. Europace 10: 668 673

29. Karasoy D, Bo Jensen T, Hansen ML, Schmiegelow M, Lamberts M, et al (2013) Obesity is a risk factor for atrial fibrillation among fertile young women: a nationwide cohort study. Europace 15: 781-786.

30. Schotten U, Verheule S, Kirchhof P, Goette A (2011) Pathophysiological mechanisms of atrial fibrillation: a translational appraisal. Physiol Rev 91: 265 325

31. Wanahita N, Messerli FH, Bangalore S, Gami AS, Somers VK, et al. (2008) Atrial fibrillation and obesity--results of a meta-analysis. Am Heart J 155: 310 315 
32. Menezes AR, Lavie CJ, DiNicolantonio JJ, O'Keefe J, Morin DP, et al. (2013) Atrial fibrillation in the 21st century: a current understanding of risk factors and primary prevention strategies. Mayo Clin Proc 88: 394-409.

33. Psaty BM, Manolio TA, Kuller LH, Kronmal RA, Cushman M, et al. (1997) Incidence of and risk factors for atrial fibrillation in older adults. Circulation 96: 2455-2461.

34. Tsang TS, Barnes ME, Miyasaka Y, Cha SS, Bailey KR, et al. (2008) Obesity as a risk factor for the progression of paroxysmal to permanent atrial fibrillation: a longitudinal cohort study of 21 years. Eur Heart J 29: 2227-2233.

35. Abed HS, Wittert GA (2013) Obesity and atrial fibrillation. Obes Rev 14: 929938.

36. Gersh BJ, Tsang TS, Barnes M, Seward JB (2005) The changing epidemiology and natural history of nonvalvular atrial fibrillation: the role of novel risk factors. Eur Heart J Suppl 7: C5-C11.

37. Wang TJ, Parise H, Levy D, D'Agostino RB Sr, Wolf PA, et al. (2004) Obesity and the risk of new-onset atrial fibrillation. JAMA 292: 2471-2477.

38. Munger TM, Dong YX, Masaki M, Oh JK, Mankad SV, et al. (2012) Electrophysiological and hemodynamic characteristics associated with obesity in patients with atrial fibrillation. J Am Coll Cardiol 60: 851-860.

39. McCully BH, Hasan W, Streiff CT, Houle JC, Woodward WR, et al. (2013) Sympathetic cardiac hyperinnervation and atrial autonomic imbalance in dietinduced obesity promote cardiac arrhythmias. Am J Physiol Heart Circ Physiol 305: H1530-1537.

40. Aimé-Sempé C, Folliguet T, Rücker-Martin C, Krajewska M, Krajewska S, et al. (1999) Myocardial cell death in fibrillating and dilated human right atria. J Am Coll Cardiol 34: 1577-1586.

41. Polontchouk L, Haefliger JA, Ebelt B, Schaefer T, Stuhlmann D, et al. (2001) Effects of chronic atrial fibrillation on gap junction distribution in human and rat atria. J Am Coll Cardiol 38: 883-891.

42. Allessie M, Ausma J, Schotten U (2002) Electrical, contractile and structural remodeling during atrial fibrillation. Cardiovasc Res 54: 230-246.

43. Lavie CJ, Amodeo C, Ventura HO, Messerli FH (1987) Left atrial abnormalities indicating diastolic ventricular dysfunction in cardiopathy of obesity. Chest 92 : 1042-1046.

44. Vaziri SM, Larson MG, Lauer MS, Benjamin EJ, Levy D (1995) Influence of blood pressure on left atrial size. The Framingham Heart Study. Hypertension 25: $1155-1160$

45. Wong CY, O'Moore-Sullivan T, Leano R, Byrne N, Beller E, et al. (2004) Alterations of left ventricular myocardial characteristics associated with obesity. Circulation 110: 3081-3087.

46. Al Chekakie MO, Welles CC, Metoyer R, Ibrahim A, Shapira AR, et al. (2010) Pericardial fat is independently associated with human atrial fibrillation. J Am Coll Cardiol 56: 784-788.

47. Perez MV, Wang PJ, Larson JC, Soliman EZ, Limacher M, et al. (2013) Risk factors for atrial fibrillation and their population burden in postmenopausal women: the Women's Health Initiative Observational Study. Heart 99: 11731178.

48. Tedrow UB, Conen D, Ridker PM, Cook NR, Koplan BA, et al. (2010) The longand short-term impact of elevated body mass index on the risk of new atrial fibrillation the WHS (women's health study). J Am Coll Cardiol 55: 2319-2327.

49. Curhan GC, Chertow GM, Willett WC, Spiegelman D, Colditz GA, et al. (1996) Birth weight and adult hypertension and obesity in women. Circulation 94: 1310-1315.

50. Conen D, Tedrow UB, Cook NR, Buring JE, Albert CM (2010) Birth weight is a significant risk factor for incident atrial fibrillation. Circulation 122: 764-770.

51. Hernandez AV, Kaw R, Pasupuleti V, Bina P, loannidis JP, et al. (2013) Association between obesity and postoperative atrial fibrillation in patients undergoing cardiac operations: a systematic review and meta-analysis. Ann Thorac Surg 96: 1104-1116.

52. Badheka AO, Rathod A, Kizilbash MA, Garg N, Mohamad T, et al. (2010) Influence of obesity on outcomes in atrial fibrillation: yet another obesity paradox. Am J Med 123: 646-651.

53. Seyfeli E, Duru M, Kuvandik G, Kaya H, Yalcin F (2006) Effect of obesity on
P-wave dispersion and QT dispersion in women. Int J Obes (Lond) 30: 957961.

54. Baranchuk A (2012) Sleep apnea, cardiac arrhythmias, and conduction disorders. J Electrocardiol 45: 508-512.

55. Guzzardi MA, lozzo P (2011) Fatty heart, cardiac damage, and inflammation. Rev Diabet Stud 8: 403-417.

56. Lin YK, Chen YC, Chang SL, Lin YJ, Chen JH, et al. (2013) Heart failure epicardial fat increases atrial arrhythmogenesis. Int J Cardiol 167: 1979-1983.

57. Chang LC, Huang KC, Wu YW, Kao HL, Chen CL, et al. (2009) The clinical implications of blood adiponectin in cardiometabolic disorders. J Formos Med Assoc 108: 353-366.

58. Conen D, Ridker PM, Everett BM, Tedrow UB, Rose L, et al. (2010) A multimarker approach to assess the influence of inflammation on the incidence of atrial fibrillation in women. Eur Heart J 31: 1730-1736.

59. Musaad S, Haynes EN (2007) Biomarkers of obesity and subsequent cardiovascular events. Epidemiol Rev 29: 98-114.

60. Lin YK, Chen YJ (2013) Adipocitokines modulate ionic currents - A key to lipotoxicity potentiated cardiac arrhythmia. J Arrhythmia 29: 247-248.

61. Frustaci A, Chimenti C, Bellocci F, Morgante E, Russo MA, et al. (1997) Histological substrate of atrial biopsies in patients with lone atrial fibrillation. Circulation 96: 1180-1184.

62. Nakamura $\mathrm{Y}$, Nakamura $\mathrm{K}$, Fukushima-Kusano $\mathrm{K}$, Ohta $\mathrm{K}$, Matsubara $\mathrm{H}$, et al. (2003) Tissue factor expression in atrial endothelia associated with nonvalvular atrial fibrillation: possible involvement in intracardiac thrombogenesis. Thromb Res 111: 137-142.

63. Aviles RJ, Martin DO, Apperson-Hansen C, Houghtaling PL, Rautaharju P, et al. (2003) Inflammation as a risk factor for atrial fibrillation. Circulation 108: 3006-3010.

64. Mano Y, Anzai T, Kaneko H, Nagatomo Y, Nagai T, et al. (2011) Overexpression of human C-reactive protein exacerbates left ventricular remodeling in diabetic cardiomyopathy. Circ J 75: 1717-1727.

65. Shimano M, Shibata R, Tsuji Y, Kamiya H, Uchikawa T, et al. (2008) Circulating adiponectin levels in patients with atrial fibrillation. Circ J 72: 1120-1124.

66. Lin YK, Chen YJ, Chen SA (2010) Potential atrial arrhythmogenicity of adipocytes: implications for the genesis of atrial fibrillation. Med Hypotheses 74: 1026-1029.

67. Nagashima K, Nakahara S, Okumura Y, Mano H, Sonoda K, et al. (2013) Termination of atrial fibrillation by ablation of high-dominant frequency sites adjacent to epicardial adipose tissue. J Arrhythm 29: 242-243.

68. Lin YK, Lai MS, Chen YC, Cheng CC, Huang JH, et al. (2012) Hypoxia and reoxygenation modulate the arrhythmogenic activity of the pulmonary vein and atrium. Clin Sci (Lond) 122: 121-132.

69. Lin YK, Chen YC, Chen JH, Chen SA, Chen YJ (2012) Adipocytes modulate the electrophysiology of atrial myocytes: implications in obesity-induced atrial fibrillation. Basic Res Cardiol 107: 293.

70. Duflou J, Virmani R, Rabin I, Burke A, Farb A, et al. (1995) Sudden death as a result of heart disease in morbid obesity. Am Heart J 130: 306-313.

71. Kannel WB, Plehn JF, Cupples LA (1988) Cardiac failure and sudden death in the Framingham Study. Am Heart J 115: 869-875.

72. Lalani AP, Kanna B, John J, Ferrick KJ, Huber MS, et al. (2000) Abnormal signal-averaged electrocardiogram (SAECG) in obesity. Obes Res 8: 20-28.

73. Chugh SS, Kelly KL, Titus JL (2000) Sudden cardiac death with apparently normal heart. Circulation 102: 649-654.

74. Eisenstein EL, Shaw LK, Nelson CL, Anstrom KJ, Hakim Z, et al. (2002) Obesity and long-term clinical and economic outcomes in coronary artery disease patients. Obes Res 10: 83-91.

75. du Toit EF, Smith W, Muller C, Strijdom H, Stouthammer B, et al. (2008) Myocardial susceptibility to ischemic-reperfusion injury in a prediabetic mode of dietary-induced obesity. Am J Physiol Heart Circ Physiol 294: H2336-2343.

76. Aubin MC, Cardin S, Comtois P, Clément R, Gosselin H, et al. (2010) A high-fat diet increases risk of ventricular arrhythmia in female rats: enhanced arrhythmic risk in the absence of obesity or hyperlipidemia. J Appl Physiol (1985) 108: 933940. 
77. Breijo-Marquez FR (2012) Cardiac Arrhythmias-New Considerations. InTech, Rijeka, Croatia.

78. Mittendorfer B, Peterson LR (2008) Cardiovascular Consequences of Obesity and Targets for Treatment. Drug Discov Today Ther Strateg 5: 53-61.

79. Bagi Z (2009) Mechanisms of coronary microvascular adaptation to obesity. Am J Physiol Regul Integr Comp Physiol 297: R556-567.

80. Barbosa JA, Rodrigues AB, Mota CC, Barbosa MM, Simões e Silva AC (2011) Cardiovascular dysfunction in obesity and new diagnostic imaging techniques: the role of noninvasive image methods. Vasc Health Risk Manag 7: 287-295.

81. Lee KT, Tang PW, Tsai WC, Liu IH, Yen HW, et al. (2013) Differential effects of central and peripheral fat tissues on the delayed rectifier $\mathrm{K}(+)$ outward currents in cardiac myocytes. Cardiology 125: 118-124.

82. Schunkert H (2002) Obesity and target organ damage: the heart. Int J Obes Relat Metab Disord 26 Suppl 4: S15-20.

83. Alpert MA, Terry BE, Cohen MV, Fan TM, Painter JA, et al. (2000) The electrocardiogram in morbid obesity. Am J Cardiol 85: 908-910, A10.

84. Fraley MA, Birchem JA, Senkottaiyan N, Alpert MA (2005) Obesity and the electrocardiogram. Obes Rev 6: 275-281.

85. Zipes DP, Camm AJ, Borggrefe M, Buxton AE, Chaitman B, et al. (2006) ACC/AHA/ESC 2006 Guidelines for management of patients with ventricular arrhythmias and the prevention of sudden cardiac death - executive summary. Eur Heart J 27: 2099-2140.

86. Ramirez AH, Schildcrout JS, Blakemore DL, Masys DR, Pulley JM, et al. (2011) Modulators of normal electrocardiographic intervals identified in a large electronic medical record. Heart Rhythm 8: 271-277.

87. SimÅŸek H, DoÄŸan A, Sahin M, GÃ 1/4mr $\tilde{A}^{1} 1 / 4 k A \tilde{A} \tilde{A}^{1} 1 / 40 A ̈$ Ÿlu HA (2013) [A case of idiopathic ventricular tachycardia in a 14-year-old obese patient due to golden berry fruit extract pills for weight loss]. Turk Kardiyol Dern Ars 41: 429-432.

88. Pareek M, Pedersen RL, Leren TP, Jensen HK (2013) [Weight loss pills purchased on the internet as the cause of ventricular fibrillation]. Ugeskr Laeger 175: 739-740.

89. Ernest D, Gershenzon A, Corallo CE, Nagappan R (2008) Sibutramineassociated QT interval prolongation and cardiac arrest. Ann Pharmacother 42: 1514-1517.

90. Schamroth CL (2012) The perils of pharmacological treatment for obesity: a case of sibutramine-associated cardiomyopathy and malignant arrhythmias. Cardiovasc J Afr 23: e11-12.

91. Peiris AN, Thakur RK, Sothmann MS, Gustafson AB, Hennes MI, et al. (1991) Relationship of regional fat distribution and obesity to electrocardiographic parameters in healthy premenopausal women. South Med J 84: 961-965.

92. el-Gamal A, Gallagher D, Nawras A, Gandhi P, Gomez J, et al. (1995) Effects of obesity on QT, RR, and QTc intervals. Am J Cardiol 75: 956-959.

93. Corbi GM, Carbone S, Ziccardi P, Giugliano G, Marfella R, et al. (2002) FFAs and QT intervals in obese women with visceral adiposity: effects of sustained weight loss over 1 year. J Clin Endocrinol Metab 87: 2080-2083.

94. Benoit SR, Mendelsohn AB, Nourjah P, Staffa JA, Graham DJ (2005) Risk factors for prolonged QTc among US adults: Third National Health and Nutrition Examination Survey. Eur J Cardiovasc Prev Rehabil 12: 363-368.

95. Ozmen N, Cebeci BS, Kardesoglu E, Cincik H, Cekin E, et al. (2006) QT dispersion in non-apneic simple snoring patients and the effect of surgical therapy on QT dispersion. Int J Cardiol 113: 82-85.

96. Arslan E, YiÄŸiner O, YavaÅŸoÄŸlu I, OzÃßelik F, KardeÄŸoÄŸlu E, et al. (2010) Effect of uncomplicated obesity on QT interval in young men. Pol Arch Med Wewn 120: 209-213.

97. Girola A, Enrini R, Garbetta F, Tufano A, Caviezel F (2001) QT dispersion in uncomplicated human obesity. Obes Res 9: 71-77.

98. Nomura A, Zareba W, Moss AJ (2000) Obesity does not influence electrocardiographic parameters in coronary patients. Am J Cardiol 85: 106108, A9.

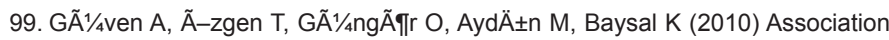
between the corrected QT interval and carotid artery intima-media thickness in obese children. J Clin Res Pediatr Endocrinol 2: 21-27.

100. Soydinc S, Davutoglu V, Akcay M (2006) Uncomplicated metabolic syndrome is associated with prolonged electrocardiographic QTC interval and QTC dispersion. Ann Noninvasive Electrocardiol 11: 313-317.

101. Marfella R, De Angelis L, Nappo F, Manzella D, Siniscalchi M, et al. (2001) Elevated plasma fatty acid concentrations prolong cardiac repolarization in healthy subjects. Am J Clin Nutr 73: 27-30.

102. Grekin RJ, Vollmer AP, Sider RS (1995) Pressor effects of portal venous oleate infusion. A proposed mechanism for obesity hypertension. Hypertension 26 193-198.

103. Mizia-Stec K, Mandecki T, Zahorska-Markiewicz B, Szulc A, Jastrzebska-Maj E, et al. (2000) [The QT interval dispersion and ventricular late potential in obese women]. Pol Merkur Lekarski 8: 84-86.

104. Mukerji R, Petruc M, Fresen JL, Terry BE, Govindarajan G, et al. (2012) Effect of weight loss after bariatric surgery on left ventricular mass and ventricular repolarization in normotensive morbidly obese patients. Am J Cardiol 110: 415-419.

105. Al-Salameh A, Allain J, Jacques A, Verhaeghe P, Desailloud R (2013) Shortening of the QT Interval is Observed Soon after Sleeve Gastrectomy in Morbidly Obese Patients. Obes Surg.

106. Pringle TH, Scobie IN, Murray RG, Kesson CM, Maccuish AC (1983) Prolongation of the QT interval during therapeutic starvation: a substrate for malignant arrhythmias. Int J Obes 7: 253-261.

107. Goldberger JJ, Cain ME, Hohnloser SH, Kadish AH, Knight BP, et al. (2008) American Heart Association/American College of Cardiology Foundation/ Heart Rhythm Society scientific statement on noninvasive risk stratification techniques for identifying patients at risk for sudden cardiac death: a scientific statement from the American Heart Association Council on Clinical Cardiology Committee on Electrocardiography and Arrhythmias and Council on Epidemiology and Prevention. Circulation 118: 1497-1518.

108. Masui A, Tsuji H, Tamura K, Tarumi N, Sugiura T, et al. (1994) Effect of body characteristics on the variables of signal-averaged electrocardiograms in healthy subjects. Chest 105: 1357-1359.

109. Aijaz B, Ammar KA, Lopez-Jimenez F, Redfield MM, Jacobsen SJ, et al. (2008) Abnormal cardiac structure and function in the metabolic syndrome: a population-based study. Mayo Clin Proc 83: 1350-1357.

110. Strbak V (2007) Joint Meeting of the Slovak Physiological Society, the Physiological Society and the Federation of European Physiological Societies. Bratislava (Slovak Republic), September 11-14, 2007. Medimond International Proceedings. Monduzzi Editore, Bologna, Italy.

111. Bharati S, Lev M (1995) Cardiac conduction system involvement in sudden death of obese young people. Am Heart J 129: 273-281.

112. Grimm W, Becker HF (2006) Obesity, sleep apnea syndrome, and rhythmogenic risk. Herz 31: 213-218.

113. Goyal SK, Sharma A (2013) Atrial fibrillation in obstructive sleep apnea. World J Cardiol 5: 157-163.

114. Khositseth A, Nantarakchaikul P, Kuptanon T, Preutthipan A (2011) QT dispersion in childhood obstructive sleep apnoea syndrome. Cardiol Young 21: $130-135$.

115. Kales SN, Straubel M (2013) Obstructive Sleep Apnea in North American Commercial Drivers. Ind Health.

116. Barreiro B, Garcia L, Lozano L, Almagro P, Quintana S, et al. (2013) Obstructive sleep apnea and metabolic syndrome in spanish population. Open Respir Med J 7: 71-76.

117. Namtvedt SK, Randby A, Einvik G, Hrubos-Strøm H, Somers VK, et al. (2011) Cardiac arrhythmias in obstructive sleep apnea (from the Akershus Sleep Apnea Project). Am J Cardiol 108: 1141-1146.

118. Chan KH, Wilcox I (2010) Obstructive sleep apnea: novel trigger and potential therapeutic target for cardiac arrhythmias. Expert Rev Cardiovasc Ther 8: 981 994.

119. Olmetti F, La Rovere MT, Robbi E, Taurino AE, Fanfulla F (2008) Nocturnal cardiac arrhythmia in patients with obstructive sleep apnea. Sleep Med 9: 475480.

120.Leung RS (2009) Sleep-disordered breathing: autonomic mechanisms and arrhythmias. Prog Cardiovasc Dis 51: 324-338.

121. Grimm W, Koehler U, Fus E, Hoffmann J, Menz V, et al. (2000) Outcome 
of patients with sleep apnea-associated severe bradyarrhythmias after continuous positive airway pressure therapy. Am J Cardiol 86: 688-692, A9.

122. Flemons WW, Remmers JE, Gillis AM (1993) Sleep apnea and cardiac arrhythmias. Is there a relationship? Am Rev Respir Dis 148: 618-621.

123. Mehra R, Benjamin EJ, Shahar E, Gottlieb DJ, Nawabit R, et al. (2006) Association of nocturnal arrhythmias with sleep-disordered breathing: The Sleep Heart Health Study. Am J Respir Crit Care Med 173: 910-916.

124. Tanigawa T, Yamagishi K, Sakurai S, Muraki I, Noda H, et al. (2006) Arterial oxygen desaturation during sleep and atrial fibrillation. Heart 92: 1854-1855.

125. Loomba RS, Arora R (2012) Obstructive sleep apnea and atrial fibrillation: a call for increased awareness and effective management. Am J Ther 19: e2124.

126. Ng CY, Liu T, Shehata M, Stevens S, Chugh SS, et al. (2011) Meta-analysis of obstructive sleep apnea as predictor of atrial fibrillation recurrence after catheter ablation. Am J Cardiol 108: 47-51.

127. Naruse Y, Tada H, Satoh M, Yanagihara M, Tsuneoka H, et al. (2013) Concomitant obstructive sleep apnea increases the recurrence of atria fibrillation following radiofrequency catheter ablation of atrial fibrillation: clinical impact of continuous positive airway pressure therapy. Heart Rhythm 10: 331 337.

128. Kanagala R, Murali NS, Friedman PA, Ammash NM, Gersh BJ, et al. (2003) Obstructive sleep apnea and the recurrence of atrial fibrillation. Circulation 107: 2589-2594.

129. Koshino Y, Satoh M, Katayose Y, Yasuda K, Tanigawa T, et al. (2008) Association of sleep-disordered breathing and ventricular arrhythmias in patients without heart failure. Am J Cardiol 101: 882-886.

130. Katritsis DG, Zareba W, Camm AJ (2012) Nonsustained ventricular tachycardia. J Am Coll Cardiol 60: 1993-2004.
131. Seppälä T, Partinen M, Penttilä A, Aspholm R, Tiainen E, et al. (1991) Sudden death and sleeping history among Finnish men. J Intern Med 229: 23-28.

132. Gami AS, Howard DE, Olson EJ, Somers VK (2005) Day-night pattern of sudden death in obstructive sleep apnea. N Engl J Med 352: 1206-1214.

133. Dursunoglu D, Dursunoglu N, Evrengül H, Ozkurt S, Kiliç M, et al. (2005) QT interval dispersion in obstructive sleep apnoea syndrome patients without hypertension. Eur Respir J 25: 677-681.

134. Becker HF, Koehler U, Stammnitz A, Peter JH (1998) Heart block in patients with sleep apnoea. Thorax 53 Suppl 3: S29-32.

135. Sankhla M, Sharma TK, Mathur K, Rathor JS, Butolia V, et al. (2012) Relationship of oxidative stress with obesity and its role in obesity induced metabolic syndrome. Clin Lab 58: 385-392.

136. Drolet B, Simard C, Gailis L, Daleau P (2007) Ischemic, genetic and pharmacological origins of cardiac arrhythmias: the contribution of the Quebec Heart Institute. Can J Cardiol 23 Suppl B: 15B-22B.

137. Jeong EM, Liu M, Sturdy M, Gao G, Varghese ST, et al. (2012) Metabolic stress, reactive oxygen species, and arrhythmia. J Mol Cell Cardiol 52: 454 463.

138. Brown DA, Aon MA, Akar FG, Liu T, Sorarrain N, et al. (2008) Effects of 4'-chlorodiazepam on cellular excitation-contraction coupling and ischaemiareperfusion injury in rabbit heart. Cardiovasc Res 79: 141-149.

139. Wehrens XH, Lehnart SE, Marks AR (2005) Intracellular calcium release and cardiac disease. Annu Rev Physiol 67: 69-98.

140. Venetucci LA, Trafford AW, O'Neill SC, Eisner DA (2008) The sarcoplasmic reticulum and arrhythmogenic calcium release. Cardiovasc Res 77: 285-292. 